\title{
Acute Myocardial Infarction in the First Trimester of Pregnancy in a Great Grand Multiparous Woman with Poorly Controlled Chronic Hypertension
}

\author{
Lakha Prasannan, MD ${ }^{1}$ Matthew J. Blitz, MD, MBA ${ }^{2}$ \\ ${ }^{1}$ Department of Obstetrics and Gynecology, Hofstra Northwell School of \\ Medicine, Long Island Jewish Medical Center, Hempstead, New York \\ 2 Division of Maternal-Fetal Medicine, Department of Obstetrics and \\ Gynecology, Hofstra Northwell School of Medicine, North Shore \\ University Hospital, Manhasset, New York
}

\author{
jill M. Rabin, $M D^{1}$
}

\begin{abstract}
Address for correspondence Matthew J. Blitz, MD, MBA, Division of Maternal-Fetal Medicine, Department of Obstetrics and Gynecology, Hofstra Northwell School of Medicine, North Shore University Hospital, 300 Community Drive, Manhasset, NY 11030

(e-mail: mblitz@northwell.edu).
\end{abstract}

Abstract
Keywords
- myocardial infarction
- hypertension
- pregnancy
- multiparity
- advanced maternal
- age
- smoking

Introduction Acute myocardial infarction (MI) in pregnancy is a rare event, usually occurring late in gestation, either in the third trimester or in the puerperium. It is associated with significant maternal and fetal morbidity and mortality. Although diagnosis and management of $\mathrm{MI}$ in pregnancy has been discussed in the literature, management of pregnancy following an early antepartum MI, which may have more consequences for the fetus, has not received as much attention.

Case A 38-year-old great grand multiparous woman presented to the emergency department complaining of acute onset chest pain. The patient had a history of chronic hypertension and was an active smoker. She was incidentally found to be 5 weeks pregnant. She was diagnosed with an acute MI, which was treated by primary percutaneous coronary intervention. Her subsequent pregnancy course was complicated by poorly controlled chronic hypertension, but she ultimately delivered a healthy newborn at 36 weeks of gestational age.

Conclusion Good pregnancy outcomes are possible after early antepartum MI, especially with early diagnosis, appropriate treatment, and a multidisciplinary team approach to prenatal care. Delivery should occur in a tertiary referral center with experience managing high-risk obstetric patients with cardiac disease.
Acute myocardial infarction (MI) in pregnancy is a rare event with an incidence of $\sim 1$ in 10,000 to 36,000, and it is associated with significant maternal and fetal morbidity and mortality. ${ }^{1,2}$ The trend toward delayed childbearing is expected to increase the frequency of coronary artery disease (CAD) and acute coronary syndrome in pregnancy. ${ }^{3}$ Among women older than 35 years, commonly referred to as advanced maternal age, there is an increased prevalence of atherosclerotic risk factors, such as diabetes, hyperlipidemia, hypertension, obesity and metabolic syndrome. ${ }^{4}$ Women who smoke during pregnancy have an eightfold higher risk of MI. ${ }^{2}$ Pregnancy itself increases the risk nearly 4-fold with approximately two-thirds of such cases occurring in multiparous women over the age of 30 years., 5 The increased risk during gestation is thought to result from the numerous maternal physiological changes that occur including hypercoagulability, vascular dysfunction, and increased myocardial oxygen demand.

Management of MI in pregnancy has been previously discussed in the literature, ${ }^{3,5,7}$ but management of pregnancy following an early antepartum MI, which may have more consequences for the fetus, has not received as much attention. Here, we present a case of a first trimester MI in a great grand multiparous female and her subsequent pregnancy course, which was complicated by poorly controlled chronic received

March 31, 2016 accepted after revision July 1, 2016
DOI http://dx.doi.org/

10.1055/s-0036-1587321. ISSN 2157-6998.
Copyright $\odot 2016$ by Thieme Medical Publishers, Inc., 333 Seventh Avenue, New York, NY 10001, USA. Tel: +1(212) 584-4662.
License terms

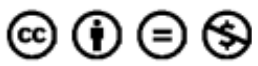


hypertension. This is followed by a discussion of critical issues and challenges in ongoing pregnancy management after a recent MI.

\section{Case}

A 38-year-old great grand multiparous African American woman, body mass index $27 \mathrm{~kg} / \mathrm{m}^{2}$, presented to the emergency department of a community hospital complaining of acute onset chest pain radiating to the shoulders bilaterally. She denied shortness of breath. The patient had a 15-year history of poorly controlled chronic hypertension and was an active smoker of approximately two packs of cigarettes per day. There was no prior history of cardiac disease, thromboembolic events, or dyslipidemia. There was also no reported family history of premature CAD. Her obstetric history included 5 term and 7 preterm deliveries, including one cesarean for breech presentation followed by 9 subsequent vaginal births. A 12-lead electrocardiogram (EKG) showed transient ST segment elevation in the inferior leads. She was incidentally found to have a serum human chorionic gonadotropin of $264 \mathrm{mIU} / \mathrm{mL}$. Transvaginal ultrasound noted a cystic structure within the uterus suggestive of an early intrauterine pregnancy. On the basis of her last menstrual period, she was 5 weeks of gestational age.

The patient was transferred to the coronary care unit of a tertiary referral center with a diagnosis of non-ST elevation MI (NSTEMI). She received aspirin, clopidogrel, heparin, metoprolol, morphine, and had persistent chest pain that was only relieved with intravenous nitroglycerin. Serial cardiac muscle enzymes demonstrated a cardiac troponin $\mathrm{T}$ (cTnT) of $0.11,0.12$, and $0.15 \mathrm{ng} / \mathrm{mL}$, and a creatine kinase-MB (CK-MB) of 3.14, 9.72, and $15.33 \mathrm{ng} / \mathrm{mL}$. Serum cholesterol levels and hemoglobin $A_{1 c}$ were normal. Echocardiography noted segmental left ventricular dysfunction with inferior and inferolateral wall hypokinesis and an ejection fraction of $49 \%$. Urgent cardiac catheterization and coronary angiogra- phy were performed after the patient was counseled about the risks and benefits, including the potential effects of ionizing radiation exposure on the developing pregnancy. Stenosis of $80 \%$ in the proximal circumflex artery was noted, along with a small filling defect consistent with a thrombus (-Fig. 1A). A successful primary percutaneous coronary intervention $(\mathrm{PCI})$ was performed and an Integrity $2.5 \times 22 \mathrm{~mm}$ bare metal stent (BMS) was implanted (-Fig. 1B; Medtronic, Minneapolis, MN). Radiation exposure to the embryo was minimized during the fluoroscopic procedure by utilizing abdominal lead shielding and limiting the total number of views (Total dose was $106 \mathrm{mGy}$.). The patient was then started on a regimen of aspirin ( $81 \mathrm{mg}$ daily), clopidogrel (75 mg daily for 1 month), and metoprolol (12.5 mg twice-daily). She was discharged in stable condition on hospital day 3 and strongly counseled for immediate cessation of smoking.

Approximately 3 weeks after the diagnosis and management of acute MI, a transvaginal ultrasound was performed for pregnancy dating. At that time, a single intrauterine gestation was noted with a crown-rump length of $1.2 \mathrm{~cm}$, corresponding to an estimated gestational age of 7 weeks and 3 days, consistent with her last menstrual period.

The patient's subsequent prenatal course was complicated by poorly controlled hypertension and multiple admissions for hypertensive urgency. She continued to smoke throughout most of her pregnancy and received inconsistent medical care due to inadequate childcare, poor transportation, and psychosocial stress. The patient frequently missed scheduled prenatal and cardiology appointments and admitted to occasional noncompliance with medications. She was admitted for blood pressure (BP) monitoring and management at $19,21,24$, and 30 weeks of gestational age. These admissions were sometimes associated with headaches. During each hospital stay, severe range BPs ( $>160 / 110$ ) were documented, and a 24-hour urine protein collection and laboratory evaluation were performed, consistently ruling out superimposed
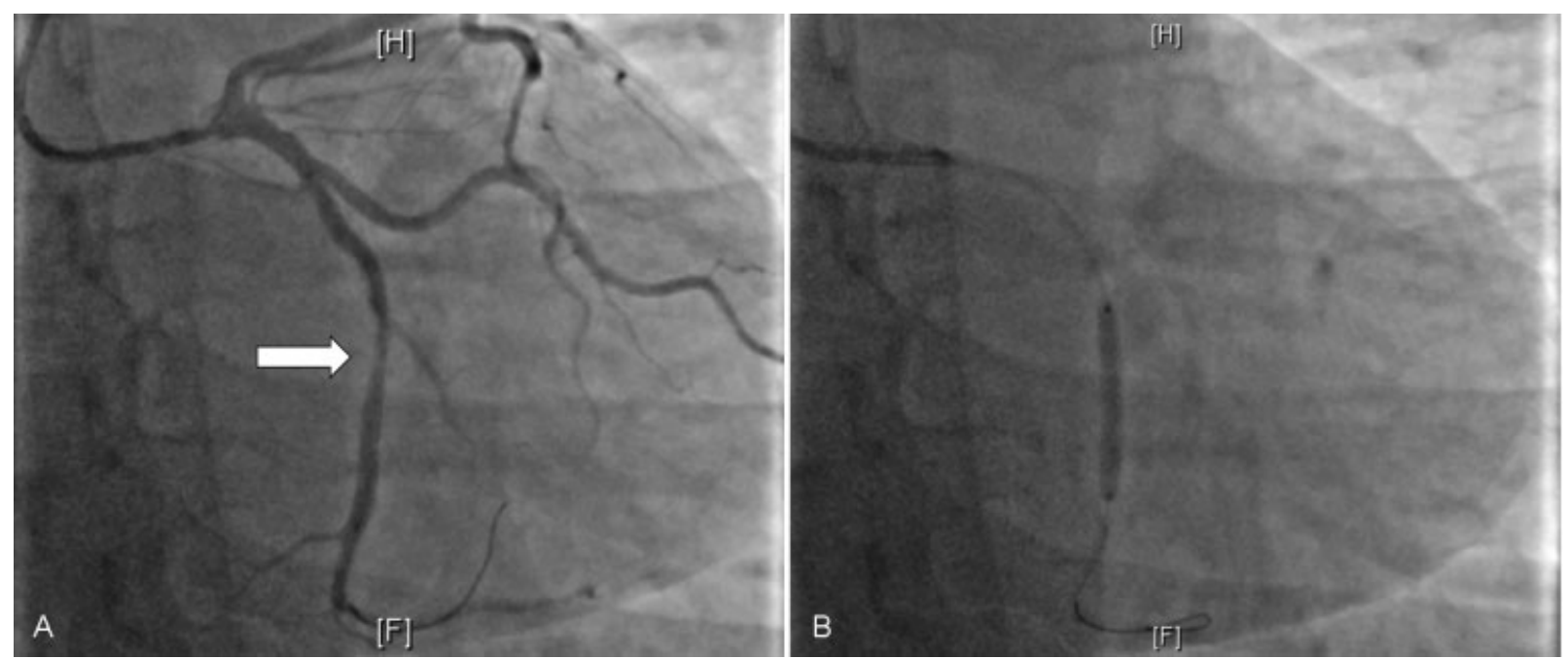

Fig. 1 Coronary angiography. (A) Stenosis of proximal circumflex artery with a small filling defect (arrow) consistent with a thrombus. (B) Successful percutaneous coronary intervention with bare metal stent placement. 
preeclampsia. At 24 weeks of gestational age, she admitted to noncompliance with the metoprolol, which was then discontinued, and she was started on methyldopa $500 \mathrm{mg}$ twice daily. She also received betamethasone for fetal lung maturity at this time, due to a history of multiple extremely preterm births ( $<28$ weeks) and her poorly controlled hypertension that may necessitate early delivery. At 30 weeks, she was started on labetalol $200 \mathrm{mg}$ twice-daily, in addition to the prior methyldopa regimen. Aspirin was continued throughout the pregnancy.

Fetal surveillance in the third trimester was consistently reassuring. Biometry demonstrated appropriate growth. Amniotic fluid volume was normal. Biophysical profiles were $8 / 8$. Nonstress tests were reactive and category 1 .

The patient was again admitted for observation at $363 / 7$ weeks of gestation for elevated BPs. Her systolic BP soon rose above $200 \mathrm{~mm} \mathrm{Hg}$, and she developed a headache. Platelet count, liver enzymes, and creatinine were normal. Urinalysis was negative for protein. She received multiple pushes of intravenous labetalol. Although the laboratory evaluation was negative, she was given a diagnosis of superimposed preeclampsia and the decision was made to induce labor based on BPs that were increasingly resistant to medical management and a new onset severe headache which was taken as evidence of end-organ dysfunction. Aspirin was discontinued. A magnesium bolus was administered. Given the increased propensity for fluid retention with magnesium, levetiracetam $500 \mathrm{mg}$ twice-daily was started for maintenance seizure prophylaxis. The patient was induced with oxytocin, received epidural anesthesia during labor, and ultimately had an uncomplicated vaginal delivery of a female newborn, weighing 2,490 g, with Apgars of 8 and 9 at 1 and 5 minutes, respectively.

The postpartum course was also complicated by severe range BPs requiring intravenous pushes of labetalol and hydralazine. Oral labetalol was increased to $400 \mathrm{mg}$ three times daily and methyldopa was increased to $500 \mathrm{mg}$ three times daily. She was discharged home on this regimen and her aspirin was resumed. The newborn had an uneventful hospital course. No malformations were observed and early postnatal development was normal.

\section{Discussion}

Although cases have been reported in all trimesters, pregnancy-associated acute MI is more common later in gestation, with three quarters occurring either in the third trimester or in the puerperium. ${ }^{6,8}$ The most common etiology, both during and outside of pregnancy, is atherosclerotic plaque rupture. ${ }^{5}$ Other etiologies include thromboembolism, coronary artery spasm and coronary artery dissection. Heart failure and cardiogenic shock are the most common maternal complications, present in nearly $40 \%$ of patients. Other complications include recurrent angina or MI (20\%), ventricular arrhythmias (12\%), and maternal death (5\%). ${ }^{2,6}$ If an MI is not recognized and not appropriately treated, maternal mortality may approach $50 \%{ }^{3}$ Most fetal deaths occur secondary to maternal death. Neonatal complications may include prematurity and low birth weight, depending on the gestational age at which the MI occurred. ${ }^{1}$
Normal pregnancy is associated with numerous maternal cardiovascular changes which collectively increase the risk of MI. Myocardial oxygen demand is increased by an expansion of circulating blood volume and a corresponding increase in cardiac output. A hypercoagulable state increases the risk of thromboembolism. In addition, labor and delivery are associated with increased hemodynamic stress. During labor, increased cardiac output occurs secondary to pain-induced catecholamine secretion, and after delivery, it occurs due to autotransfusion from the uterus and resorption of edema. ${ }^{9}$

Diagnosis of MI in pregnancy may be delayed and patients may receive suboptimal care because the presenting symptoms are often difficult to distinguish from common pregnancy ailments such as gastrointestinal reflux, musculoskeletal pain, nausea, vomiting, dizziness, and physiologic dyspnea. Furthermore, other serious conditions may occur in pregnancy such as acute pulmonary embolism and aortic dissection, which must also be ruled out. Therefore, the workup may be insufficiently thorough if ischemic heart disease is not considered. The diagnostic criteria for MI in pregnancy are essentially the same as outside of pregnancy, and include ischemic symptoms, EKG abnormalities and elevations in cardiac biomarkers. ${ }^{7}$ However, pregnancy may affect biomarker concentrations. Troponins are preferred over CK-MB, especially during labor and delivery, because uterine contractions and cell breakdown may increase myoglobin, creatine kinase and CK-MB. ${ }^{10}$

Many aspects of medical and interventional management of MI are compatible with pregnancy, but there are known risks and areas of uncertainty where limited data exists. The fetal risks associated with ionizing radiation exposure from revascularization procedures are related to the stage of pregnancy and the absorbed dose. The most sensitive period is from 8 to 15 weeks of gestation when malformations associated with the central nervous system may occur at doses above 100-200 mGy. ${ }^{11}$ Embryonic loss may occur at similar doses during the preimplantation and presomite stages, but there does not appear to be an increased risk of congenital malformations or growth restriction in surviving pregnancies. ${ }^{12}$ Although high doses of radiation (e.g., $>500$ $\mathrm{mGy}$ ) to the embryo or fetus are known to increase the risk of childhood cancer, the risk associated with exposures less than 100-200 mGy remains uncertain. ${ }^{12}$ Some medications used in the treatment of MI, such as, angiotensin-converting enzyme inhibitors and angiotensin-receptor blockers, are contraindicated in pregnancy owing to their association with fetal renal and cardiac abnormalities. Statins are also contraindicated in pregnancy based on a theoretical teratogenic risk demonstrated in some animal studies. ${ }^{13}$ Clopidogrel does not appear to be teratogenic based on animal studies and case reports. Although its safety in human pregnancy also remains uncertain, the benefits of clopidogrel are thought to outweigh the risks.

Treatment of acute MI in pregnancy should be individualized, given the potential for conflict between the needs of the mother and the fetus. A multidisciplinary team approach is recommended. Medical therapy should include antiplatelet agents (both aspirin and clopidogrel), antithrombotic agents (unfractionated heparin or enoxaparin), and $\beta$-blockers. Pain 
control can be achieved with nitrates and/or morphine. Although there may be hesitation to perform interventional procedures in obstetric patients, PCI can be safely performed. Early revascularization is essential in cases of ST elevation MI or cases of NSTEMI with recurrent ischemic symptoms or persistent EKG changes. ${ }^{7}$ The primary advantage of a BMS over a drug-eluting stent in pregnancy is that a BMS does not require long-term dual antiplatelet therapy, which may increase the risk of postpartum hemorrhage. The main disadvantage is that it is associated with a higher rate of instent restenosis. ${ }^{7}$ Systemic thrombolysis with recombinant tissue plasminogen activator (rTPA) may be performed during pregnancy if the hospital has no PCI capability and reperfusion therapy is clinically indicated, as long as there are no absolute contraindications. Although rTPA does not cross the placenta, there is a risk of retroplacental hemorrhage and placental abruption. ${ }^{14}$

There are no established guidelines for the management of pregnancy after an early antepartum MI. Outside of pregnancy, a persistently elevated systolic BP (>140 mm Hg) 6 months after an MI is associated with an increased risk of subsequent cardiovascular events. ${ }^{15}$ It was previously thought that tight control of maternal hypertension during pregnancy, using antihypertensive therapy to normalize BP, may be detrimental to the fetus, possibly contributing to fetal growth restriction. However, Magee et al recently demonstrated that there is no significant difference in the risk of adverse perinatal outcomes with tight versus less-tight control. ${ }^{16}$ Therefore, tight control of BP, which is more beneficial for the mother, may be more appropriate in pregnancy after a recent MI. In late gestation, one of the primary concerns is finding an appropriate balance between the risk of bleeding and the risk of thrombosis. If dual antiplatelet therapy is continued throughout gestation, it may be discontinued one week prior to a scheduled delivery and low-molecular-weight heparin can be initiated. However, this may not be as efficacious for the prevention of stent thrombosis. During labor and delivery, cardiac demands should be minimized. Vaginal delivery with early epidural anesthesia is preferred in most cases. Operative assistance with vacuum or forceps should be considered to reduce maternal effort. Cesarean delivery should be reserved for the usual obstetric indications and for patients with active myocardial ischemia or poor cardiac function, as defined by a significantly reduced left ventricular ejection fraction. ${ }^{8,17}$ Methylergonovine is associated with vasospasm and should be avoided in the management of postpartum hemorrhage. Non-steroidal anti-inflammatory drugs may exacerbate hypertension and should be avoided for postpartum analgesia.

The mother in this case, who had multiple atherosclerotic risk factors, experienced an NSTEMI in the first trimester of pregnancy requiring $\mathrm{PCI}$ and her subsequent prenatal course was complicated by poorly controlled chronic hypertension. She received a diagnosis of superimposed preeclampsia with severe features necessitating a medically indicated late preterm delivery. There was no evidence of fetal growth restriction. It is possible that the hormonal milieu of early pregnancy, in combination with the patient's underlying risk factors, precipitated her acute ischemic event. Nonadherence to antihypertensive therapy may have contributed to her resistant hypertension.

In summary, good pregnancy outcomes are possible after early antepartum MI, especially with early diagnosis, appropriate treatment, and a multidisciplinary team approach to prenatal care. Delivery should occur in a tertiary referral center with experience managing high-risk obstetric patients with cardiac disease.

\section{Acknowledgment}

The authors thank Stephanie Augustine for her assistance in acquiring and reviewing medical records.

\section{References}

1 Ladner HE, Danielsen B, Gilbert WM. Acute myocardial infarction in pregnancy and the puerperium: a population-based study. Obstet Gynecol 2005;105(3):480-484

2 James AH, Jamison MG, Biswas MS, Brancazio LR, Swamy GK, Myers ER. Acute myocardial infarction in pregnancy: a United States population-based study. Circulation 2006;113(12):1564-1571

3 Turitz AL, Friedman AM. Ischemic heart disease in pregnancy. Semin Perinatol 2014;38(5):304-308

4 van Katwijk C, Peeters LL. Clinical aspects of pregnancy after the age of 35 years: a review of the literature. Hum Reprod Update 1998;4(2):185-194

5 Fryearson J, Adamson DL. Heart disease in pregnancy: ischaemic heart disease. Best Pract Res Clin Obstet Gynaecol 2014;28(4): 551-562

6 Elkayam U, Jalnapurkar S, Barakkat MN, et al. Pregnancy-associated acute myocardial infarction: a review of contemporary experience in 150 cases between 2006 and 2011. Circulation 2014; 129(16):1695-1702

7 Pacheco LD, Saade GR, Hankins GD. Acute myocardial infarction during pregnancy. Clin Obstet Gynecol 2014;57(4):835-843

8 Roth A, Elkayam U. Acute myocardial infarction associated with pregnancy. J Am Coll Cardiol 2008;52(3):171-180

9 Regitz-Zagrosek V, Gohlke-Bärwolf C, Iung B, Pieper PG. Management of cardiovascular diseases during pregnancy. Curr Probl Cardiol 2014;39(4-5):85-151

10 Shade GH Jr, Ross G, Bever FN, Uddin Z, Devireddy L, Gardin JM. Troponin I in the diagnosis of acute myocardial infarction in pregnancy, labor, and post partum. Am J Obstet Gynecol 2002; 187(6):1719-1720

11 Shaw P, Duncan A, Vouyouka A, Ozsvath K. Radiation exposure and pregnancy. J Vasc Surg 2011;53(1, Suppl)28S-34S

12 Brent RL. Protection of the gametes embryo/fetus from prenatal radiation exposure. Health Phys 2015;108(2):242-274

13 Morton S, Thangaratinam S. Statins in pregnancy. Curr Opin Obstet Gynecol 2013;25(6):433-440

14 Wiese KM, Talkad A, Mathews M, Wang D. Intravenous recombinant tissue plasminogen activator in a pregnant woman with cardioembolic stroke. Stroke 2006;37(8):2168-2169

15 Thune JJ, Signorovitch J, Kober L, et al. Effect of antecedent hypertension and follow-up blood pressure on outcomes after high-risk myocardial infarction. Hypertension 2008;51(1):48-54

16 Magee LA, von Dadelszen P, Rey E, et al. Less-tight versus tight control of hypertension in pregnancy. N Engl J Med 2015;372(5): 407-417

17 Ruys TP, Roos-Hesselink JW, Pijuan-Domènech A, et al; ROPAC investigators. Is a planned caesarean section in women with cardiac disease beneficial? Heart 2015;101(7):530-536 